\title{
Studies on the spin-spin interaction between flavin and iron-sulfur cluster in an iron-sulfur flavoprotein
}

\author{
Randy C. Stevenson i* William R. Dunham " ${ }^{\text {a }}$ Richard H. Sands ", \\ Thomas P. Singer ${ }^{b}$ and Helmut Beinert ${ }^{c}$ \\ "Biophysics Research Division, Institute of Science and Technology, The University of Michigan. Ann Arbor, MI 48109. \\ ${ }^{n}$ Department of Biochemistry and Biophysics and Department of Pharmaceutical Chemistry, University of California, and \\ Molecular Biology Division, Veterans Administration Hospital, San Francisco, CA 94121, and'Institute for Enżyme \\ Research and Department of Biochemistry, College of Agricultural \& Life Sciences, University of Wisconsin, Madison, WI \\ 53706 (U.S.A.)
}

(Received June 19th, 1985)

Key words: Flavoprotein; Flavin; Spin-spin interaction; Methylamine dehydrogenase; Fe-S cluster

When the di- or trimethylamine dehydrogenases (trimethylamine:(acceptor) oxidoreductase (demethylating), EC 1.5.99.7) of certain methylotrophic bacteria are reduced by two electrons with substrate unusual EPR signals arise at $g=2$ and $g=4$ (Steenkamp, D.J. and Beinert, H. (1982) Biochem. J. 207, 233-239; 241-252) indicative of spin-spin interaction between the FMN and iron-sulfur compounds of these enzymes. An attempt is made to understand, describe and simulate these spectra in terms of a triplet state with possible contributions from both dipolar and anisotropic exchange $(J)$ interactions. No direct measurement of $J$ is available, but various approaches to setting limits to $J$ are outlined. According to these, $J \approx 0.4$ to $3 \mathrm{~cm}^{-1} \mathrm{or}^{\circ}$ 15 to $50 \mathrm{~cm}^{-1}$. The spectra show, in the $g=2$ region, a pair of rather sharp inner and a pair of broad outer lines; the latter broaden as well as move out from the center with increasing time (after substrate addition) and substrate concentration, while there is little change of $g=4$. The best fits to such spectra were obtained by assuming distribution of $D$ and $E$ values, depending on substrate effects and arriving presumably from ' $g$-strain'. The fact that both shapes and intensities at $g=2$ and $g=4$ could be reproduced simultaneously at two frequencies indicates that the assumptions underlying our approaches and interpretations are permissible and reasonable, although we cannot claim their uniqueness. The distance between the centers of the spin densities of the flavin radical and the Fe-S cluster is thought to lie between the limits 3 to $5 \AA$ if the asymmetries in the spin-spin interaction are magnetic dipole-dipole in origin. Because there is an indication that the interaction is anisotropic exchange, the upper limit is less stringent.

\section{Introduction}

We have previously reported on the purification and properties of di- and trimethylamine dehydro-

\footnotetext{
* Present address: AT \& T Bell Laboratories, 1600 Osgood St., North Andover, MA 01845, U.S.A.

Abbreviations: $W_{3} A_{1}$, bacterium $W_{3} A_{1}$ (source of TMAD); H.X, hyphomicrobium $X$ (source of TMAD and DMAD), cf. Ref. 6.
}

genases from methylotrophic bacteria [1-8]. These enzymes carry out the oxidative $N$-demethylation of di- and/or trimethylamine with the formation of formaldehyde. They are of particular interest to the student of electron transfer mechanisms insofar as they constitute the simplest examples of complex flavo- or Fe-S proteins that contain only a single flavin (FMN) and a single $[4 \mathrm{Fe}-4 \mathrm{~S}]$ cluster per subunit. Most enzymes in this class carry multiple Fe-S clusters and/or other prosthetic 
groups. Attempts to separate in time the observation of electron transfer from substrate to one of the electron acceptors in such an enzyme and of intrasubunit electron transfer between the electron carriers present has in general been unsuccessful. The methylamine dehydrogenases, however, allow a clear distinction between these processes. On addition of substrate to one of these enzymes, there is a very rapid initial two-electron transfer to the FMN prosthetic group with formation of $\mathrm{FMNH}_{2}$, which is followed by a slow one-electron transfer from $\mathrm{FMNH}_{2}$ to the Fe-S cluster. Complete three-electron reduction is not observed with substrate. This pattern becomes clearly evident through the development of a triplet state, which evidently arises through interaction between the neighboring FMNH and one-electron reduced $[4 \mathrm{Fe}-4 \mathrm{~S}]^{1+}$ cluster. EPR signals for the individual components, viz. $\mathrm{FMNH}^{\circ}$ and $[4 \mathrm{Fe}-4 \mathrm{~S}]^{1+}$, are barely detectable. On the other hand, on partial reduction with the chemical reductant, dithionite, the triplet state does not arise, but separate EPR signals indicating $\mathrm{FMNH}^{\circ}$ and a $[4 \mathrm{Fe}-4 \mathrm{~S}]^{1+}$ cluster are observed. On further reduction, $\mathrm{FMNH}_{2}$ is formed and all Fe-S cluster present is reduced. This behavior suggested that binding of substrate induces a conformational change that brings about the geometry favorable for the strong spin-spin interaction observed. This explanation is supported by experiments using a nonoxidizable substrate analog, viz. the tetramethylammonium ion, which is bound by the enzyme and induces the disposition in which the triplet state can arise also upon chemical reduction by dithionite.

While biochemical studies of the enzymes were reported previously [1-7], in this paper an attempt is made to understand the unusual EPR signals of the triplet state found in these enzymes on interaction with substrate. It was of particular help in these efforts that three enzymes of this type were available that showed qualitatively very similar but quantitatively different behavior. A preliminary account of this work has been presented at the Seventh International Symposium on Flavins and Flavoproteins, Ann Arbor, MI, June 21-26, 1981 [8].

\section{Materials and Methods}

Purification, characterization and storage of the enzymes and procedures for oxidoreductive titrations, rapid freeze-quenching experiments and routine EPR measurements were as described in previous publications $[1,2,6]$.

EPR spectra for special purposes were taken on reflection type spectrometers at temperatures between 10 and $20 \mathrm{~K}$, which were produced by helium flow systems. The S-band ( $3 \mathrm{GHz}$ ) spectrometer was first constructed and described by Strong [9] with a Sperry Rand 2K41 Klystron tube as a microwave source and a quartz-filled dielectric cavity operating in the TE102 mode. Stevenson [10] later rebuilt the cavity and added an improved AFC unit. The X-band $(9 \mathrm{GHz})$ spectra were taken on a standard Varian Century Line spectrometer. The P-band $(15 \mathrm{GHz})$ spectra were taken on a spectrometer first constructed by Reid [11] for ENDOR studies. Its microwave source is a Varian experimental klystron tube; its cavity is a frequency-tunable cylindrical cavity operating in the TE011 mode and was constructed and described by Stevenson [10].

Usually, the analog output of the spectrometers was recorded digitally on a TRACOR/Northern NS-570 signal averager, then transmitted to a DEC VAX $11 / 750$ where the data were reduced to Hamiltonian parameters by simulating the signals with the computer program TRIPLET that was designed to be the most general possible for an $S=1$ spin system $[10,12]$. The Hamiltonian for this program is

$$
\begin{aligned}
H= & \vec{S} \cdot \dot{G} \cdot \vec{B}+R^{+}(\alpha, \beta, \gamma)\left\{D\left[S^{2}-\frac{S(S+1)}{3}\right]\right. \\
& \left.+E\left[S_{x}^{2}-S_{y}^{2}\right]\right\} R(\alpha, \beta, \gamma)
\end{aligned}
$$

where $\tilde{G}$ is the g-tensor, with three degrees of freedom, $g_{x}, g_{y}$ and $g_{z}$, and $D$ and $E$ are the zero-field splitting parameters. These latter parameters, together with the Euler angles specifying the relative orientations of the $g$-tensor principal axis system and the frame of the zero-field splitting tensor, $\alpha, \beta$, and $\gamma$, make up the five degrees of freedom in the $D_{2}$ representation [12] of the zerofield Hamiltonian. Thus, there are a total of eight degrees of freedom in the Hamiltonian, out of the 
nine possible for a spin one system; the scalar term is not measurable by EPR. Therefore, the Hamiltonian has all the generality possible for a spin one system. However, we need to qualify this claim because the analysis showed that $D$ and $E$ must necessarily be distributed variables to obtain good computer fits to the data for tri- and dimethylamine dehydrogenases.

With an assumption that $D$ and $E$ are distributed variables, multivariate statistical analysis is necessary to describe these variables correctly. Because the computation cost for a complete analysis is prohibitive, we assumed $D$ and $E$ to be independent, normally distributed, random variables. These assumptions led to acceptable fits, so that we did not make more complex assumptions nor did we test the uniqueness of the assumptions. Because $D$ and $E$ are distributed, one-spin Hamiltonian cannot represent the physical system. Therefore, the $g$-values and zero-field splitting parameters must be considered as descriptors of an ensemble's average tendency rather than properties of a unique physical system. For EPR, the observables are the position, intensity and linewidth of a transition. If ' $G$-strain', ' $D$-strain' or ' $J$-strain' is present (indicating protein microheterogeneties which are either locked-in by freezing or caused by freezing [13]), then these observables are functions of random variables. The moments of these observables are theoretically calculable via multivariate analysis, although we did not attempt such a calculation. However, from similar calculations on other Hamiltonians [13] we are convinced that our use of $G$-tensors in the spectral simulations of TMAD and DMAD is sufficiently accurate for the purposes of this work. Some specific applications of strain theory distributions are considered in the conclusions.

The details of the simulation program TRIPLET along with several procedures for fitting multi-frequency EPR data have been published elsewhere $[10,12]$. The simulation program is for triplet EPR spectra. In this case, the use of this program implies that, $J$, the scalar coupling constant, is much larger than $\left\|\tilde{G}_{1}-\tilde{G}_{2}\right\| \beta B$ where $\tilde{G}_{1}$ and $\tilde{G}_{2}$ are the $G$-tensors for the two interacting $S=1 / 2$ systems that give rise to the triplet. The importance of this bound will be discussed further after we have presented the results of our fitting efforts.

\section{Results}

As mentioned in the introduction, oxidized trimethylamine dehydrogenase can be fully reduced by three electron equivalents of dithionite. The result is a fully reduced, diamagnetic $\mathrm{FMNH}_{2}$ moiety and an $S=1 / 2[4 \mathrm{Fe}-4 \mathrm{~S}]^{1+(2+; 1+)}$ center. The X-band $(9 \mathrm{GHz})$ spectrum of the fully reduced trimethylamine dehydrogenase is shown in Fig. 1. In the early stages of titration by dithionite, spectrometer conditions can be optimized to display the semiquinone state of FMN as a single line at $g=2.005$ with a $17 \mathrm{G}$ linewidth at X-band. Upon titration with substrate, the trimethylamine dehydrogenase from $W_{3} A_{1}$ has the EPR spectrum shown in Fig. 2, this spectrum is different from either of that shown in Fig. 1 or that of the semiquinone, although small amounts of both are visible in Fig. 2a. For the moment, we shall ignore the questions regarding possible conformation changes associated with this signal and focus only on the physical interpretation of the signal itself.

Our first reaction to seeing this signal was that it resulted from the interaction between the reduced $\mathrm{Fe}-\mathrm{S}$ center and the semiquinone state of the FMN molecule, since the overall appearance of the signal was similar to EPR interaction signals that we had seen before $[14,15]$. It was also clear

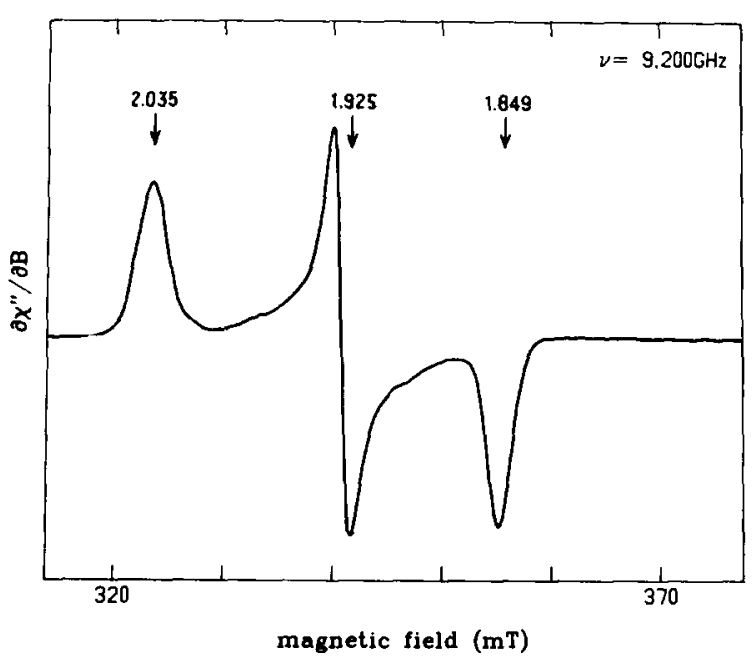

Fig. 1. The X-band EPR spectrum of fully reduced trimethylamine dehydrogenase from $W_{3} A_{1}$ at $15 \mathrm{~K}$. Spectrometer settings: frequency, $9.200 \mathrm{GHz}$; power, $1 \mathrm{~mW}$; modulation amplitude, $10 \mathrm{G}$; sweep time, $240 \mathrm{~s}$; time constant, $0.128 \mathrm{~s}$. 


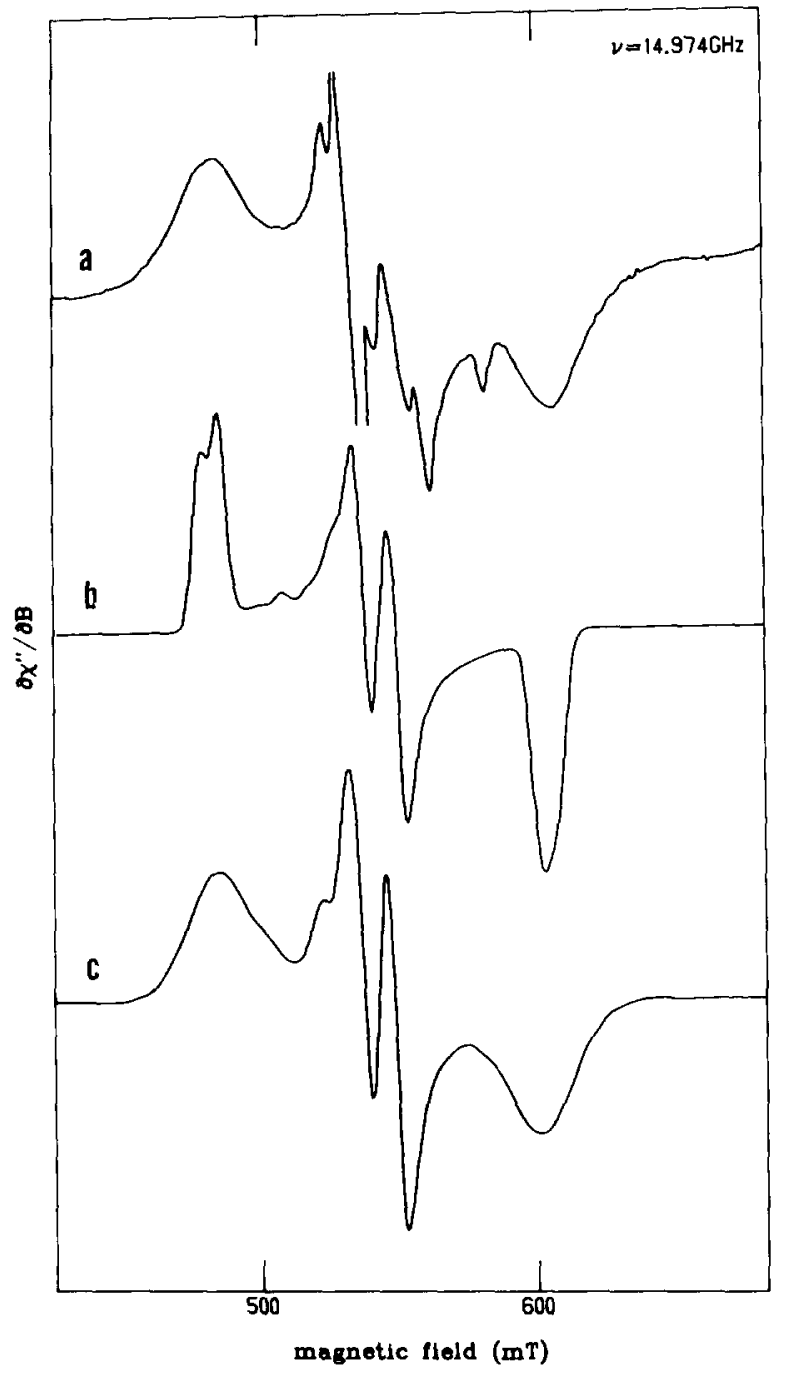

Fig. 2. The g-2 region of the P-band EPR spectrum of substrate reduced trimethylamine dehydrogenase from $W_{3} A_{1}$ at $18 \mathrm{~K}$. (a) Experimental spectrum; (b) simulation without zero-field splitting distribution; (c) simulation with zero-field splitting distribution. Spectrometer settings: frequency, $14.974 \mathrm{GHz}$; power, $0.2 \mathrm{~mW}$; modulation amplitude, $15 \mathrm{G}$; sweep time, $900 \mathrm{~s}$; time constant, 1 s. See Table I for simulation parameters.

that the interaction was much stronger than we had seen previously. Since both of the interacting spin systems are $S=1 / 2$, then we might expect our previous computer program, RASCAL [16], to approach a good fit. When these fitting attempts failed, we decided to write a program for 'strong interactions', called TRIPLET [12], which would calculate the EPR spectra from an $S=1$ system.
We assumed that, in this case, the $S=1$ state was formed by an exchange interaction between FMN and $[4 \mathrm{Fe}-4 \mathrm{~S}]$. A P-band simulation is shown in Fig. 2b.

The simulation has merit; however, it is not satisfactory for several reasons that mainly concern the 'half-field' or $g=4$ resonance shown in Fig. 3a (simulation in Fig. 3b). There are many parameters in these fits and although the problem of fitting triplet spectra is formidable, the parameters are uniquely determined by virtue of the interplay between the fitting of the $g=4$ and $g=2$ regions.

For instance, the $g=4$ region is very sensitive to changes in the g-values, linewidths, and Euler

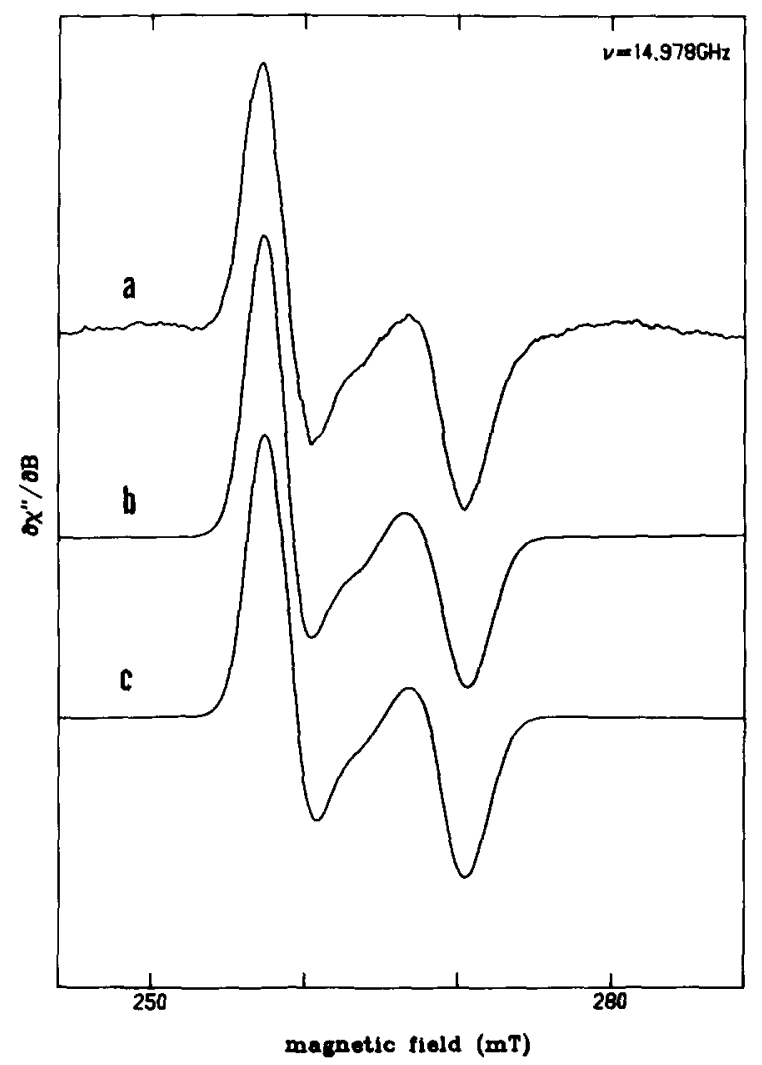

Fig. 3. The $g=4$ region of the P-band EPR spectrum of substrate reduced trimethylamine dehydrogenase from $W_{3} A_{1}$ at 29 K. (a) Experimental spectrum; (b) simulation without zerofield splitting distribution; (c) simulation with zero-field splitting distribution. Spectrometer settings: frequency, $24.978 \mathrm{GHz}$; power, $4 \mathrm{~mW}$; modulation amplitude, $15 \mathrm{~g}$; sweep time, $900 \mathrm{~s}$; time constant, $1 \mathrm{~s}$. See Table I for simulation parameters. 
angles, but less sensitive than the $g=2$ region in the $D$ and $E$ values. By using this selective sensitivity to parameters, we can refine the fits and thereby determine the parameter values by alternating between the two spectra. Figs. $2 b$ and $3 b$ represent the result of this procedure, the parameters having been uniquely and accurately determined. However, as one can see by comparing Fig. 2a with Fig. 2b, the linewidths in the simulated spectra for the outer resonances in the $g=2$ region seem to be too small to allow a good fit. The resonances in the $g=4$ and the $g=2$ regions are merely different transitions between the same three states; therefore, the linewidth of the $g=2$ region is not a free parameter and must be about twice that of the $g=4$ region. Apparently, then, these fits show that the two spectra cannot be fit by the same parameters. For this reason and the existence of $g$-strain as discussed in the section on Conclusions, we introduced a distribution in the values for the parameters, $D$ and $E$. In Figs. 2c and $3 c$, we show the fits after the addition of the distribution in the zero-field splitting parameters. To match the data, the distribution had to keep the inner lines at $g=2$ narrow while broadening the outer lines. Therefore, we chose the variance, $\delta D$, in $D$ to satisfy the condition, $\delta D / D=$ constant; i.e., the larger the zero-field splitting, the larger the variance. The reason that the distribution does not broaden the $g=4$ linewidths is that even though the intensity of the $g=4$ resonance is

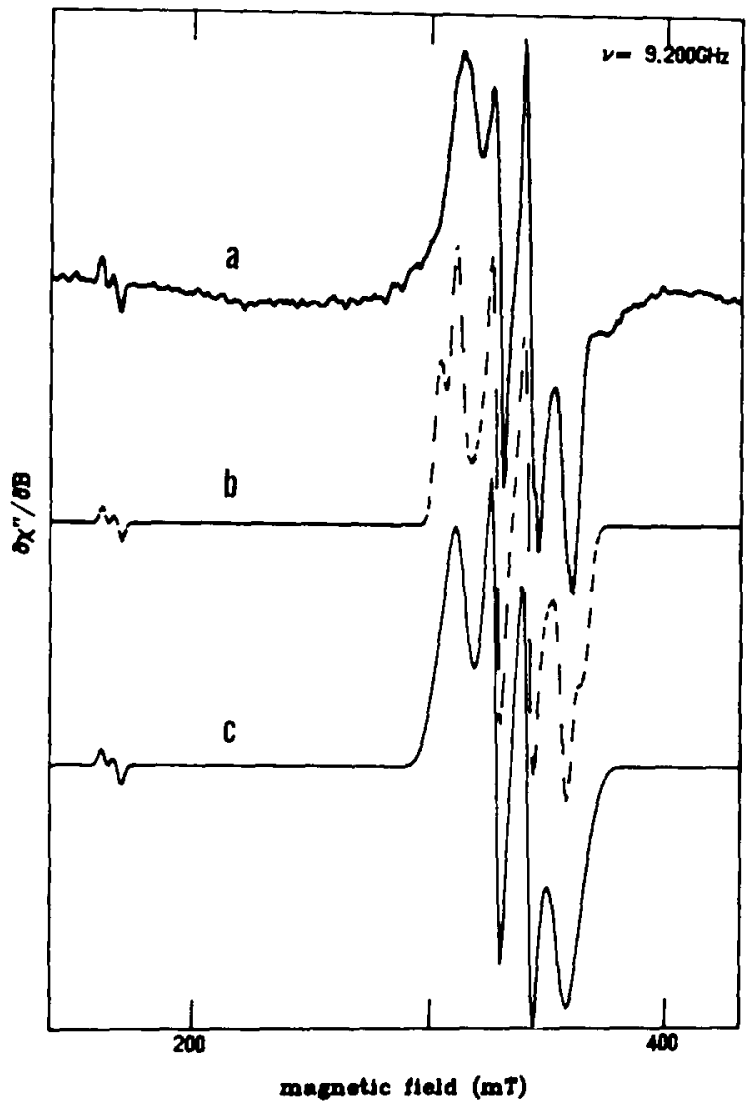

Fig. 4. The X-band EPR spectrum of substrate-reduced trimethylamine dehydrogenase from H.X at $20 \mathrm{~K}$. (a) Experimental spectrum; (b) simulation without zero-field splitting distribution; (c) simulation with zero-field splitting distribution. Spectrometer settings: frequency, $9.200 \mathrm{GHz}$; power, $40 \mathrm{~mW}$; modulation amplitude, 8 gauss; sweep time, $240 \mathrm{~s}$; time constant, $0.25 \mathrm{~s}$. See Table for simulation parameters.

Fig. 5. The X-band EPR spectrum of substrate-reduced dimethylamine dehydrogenase from H.X at $20 \mathrm{~K}$. Other details as for Fig. 4. 
TABLE I

\section{SPECTRAL FITTING PARAMETERS}

The $\sigma_{x}, \sigma_{y}, \sigma_{z}$ values are linewidths referring to the standard deviations in Gaussian lineshapes.

\begin{tabular}{lccc}
\hline Sample & $\begin{array}{l}\text { Trimethylamine } \\
\text { dehydrogenase } \\
\left(\mathrm{W}_{3} \mathrm{~A}_{1}\right)\end{array}$ & $\begin{array}{l}\text { Trimethylamine } \\
\text { dehydrogenase } \\
(\mathrm{H} . \mathrm{X} .)\end{array}$ & $\begin{array}{l}\text { Dimethylamine } \\
\text { dehydrogenase } \\
(\mathrm{H} . \mathrm{X} .)\end{array}$ \\
\hline$G_{x}$ & 1.927 & 1.927 & 1.921 \\
$G_{y}$ & 1.962 & 1.960 & 1.958 \\
$G_{z}$ & 2.020 & 2.015 & 2.015 \\
$D$ & $62.3 \mathrm{mT}$ & $42.8 \mathrm{mT}$ & $28.7 \mathrm{mT}$ \\
$E$ & $16.9 \mathrm{mT}$ & $12.7 \mathrm{mT}$ & $5.4 \mathrm{mT}$ \\
$\alpha$ & 0 & 0 & 0 \\
$\beta$ & $45^{\circ}$ & $45^{\circ}$ & $45^{\circ}$ \\
$\gamma$ & $60^{\circ}$ & $60^{\circ}$ & $60^{\circ}$ \\
$\sigma_{x}$ & $2.5 \mathrm{mT}$ & $2.1 \mathrm{mT}$ & $2.5 \mathrm{mT}$ \\
$\sigma_{y}$ & $2.7 \mathrm{mT}$ & $2.5 \mathrm{mT}$ & $1.5 \mathrm{mT}$ \\
$\sigma_{z}$ & $3.3 \mathrm{mT}$ & $2.1 \mathrm{mT}$ & $2.5 \mathrm{mT}$ \\
\hline
\end{tabular}

greatly affected by the size of $D$ and $E$, the $g=4$ line positions are not.

The high quality of the fits (Figs. $2 \mathrm{c}$ and $3 \mathrm{c}$ ), the fact that these parameters also produce high quality fits to the data taken at other frequencies (not shown), the fact that the parameters are similar in all three protein preparations we have studied (see Figs. 4 and 5), and the overdetermined nature of this spectral fitting procedure convince us that our parameters are correct. The parameters are summarized in Table $I$.

\section{Conclusions}

When an $S=1$ system results from the spin coupling of two $S=1 / 2$ systems, the algebra of the angular momentum operators gives us the relationship that

$\tilde{G}=\left(\tilde{G}_{1}+\tilde{G}_{2}\right) / 2$

where $\tilde{G}_{1}, \tilde{G}_{2}$ are the $\tilde{G}$-tensors of the interacting systems, and $\tilde{G}$ refers to the resultant spin system. If we hypothesize that the two interacting spin systems are the flavin semiquinone and [4Fe-4S] cluster, then we can write the $\tilde{G}$-tensors of these two interactions in their principal axis systems as

$\tilde{G}_{1}=G_{\mathrm{FMNH}}=2.0045\left(\begin{array}{ccc}1 & 0 & 0 \\ 0 & 1 & 0 \\ 0 & 0 & 1\end{array}\right)$
$\tilde{G}_{2}=G_{[4 \mathrm{Fe}-4 \mathrm{~S}]^{\cdot}}=\left(\begin{array}{lll}1.848 & 0 & 0 \\ 0 & 1.925 & 0 \\ 0 & 0 & 2.035\end{array}\right)$

then

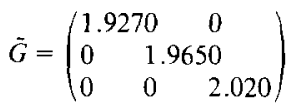

The $\tilde{G}_{1}, \tilde{G}_{2}$ and $\tilde{G}$ tensors are automatically diagonal in the same coordinate reference frame because the $\tilde{G}_{1}$ tensor is isotropic and therefore diagonal in all frames. These values are very close to the values in Table I. We conclude that the triplet is formed by the exchange coupling of the $\mathrm{FMNH}^{\circ}$ and [4Fe-4S] cluster in each of the enzymes subunits. The interaction of two FMNH radicals or two $[4 \mathrm{Fe}-4 \mathrm{~S}]^{1+}$ clusters will not give rise to the above $g$-tensor. The inter-subunit interaction of $\mathrm{FMNH}^{\circ}$ and $[4 \mathrm{Fe}-4 \mathrm{~S}]^{1+}$, possible in $\mathrm{W}_{3} \mathrm{~A}_{1}$, is not expected to produce a coupling strong enough to cause a triplet EPR spectrum, but instead a weak interaction signal such as those shown in some EPR spectra from coenzyme $B_{12}[14]$ and succinate: $Q$ oxidoreductase [15]; however, the interaction between the $\mathrm{FMNH}^{\circ}$ and the $[4 \mathrm{Fe}-4 \mathrm{~S}]^{1+}$ clusters in these proteins is large enough to form a triplet (see below), but subtle enough to leave the $g$-tensors of the interacting spin systems essentially unchanged from the states when they are EPRvisible as isolated spin systems. The meaning of this conclusion is unknown for the FMNH free radical, but for the $[4 \mathrm{Fe}-4 \mathrm{~S}]$ cluster it probably means that there is no ligand substitution or massive change in ligand position at the [4Fe-4S] site.

The triplet states in Figs. 2-5 are, therefore, caused by a nearly isotropic spin-spin interaction:

$H=-2 J \overrightarrow{S_{1}} \cdot \vec{S} 2$

We have not made a direct measurement of $J$, but since we have demonstrated the necessity of its existence, we can use our data to estimate and

- place constraints on its size. The magnitude of $J$ must be large enough to cause our fitting program to be valid and Eqn. 2 to be accurate. Mathematically, this condition reduces to $J \gg\left\|\tilde{G}_{1}-\tilde{G}_{2}\right\| \beta B$, which in turn means that $J>0.4 \mathrm{~cm}^{-1}$ when we consider the $15 \mathrm{GHz}$ EPR fits. The failure of our attempts to determine $J$ by observing EPR signal 
strengths from 4 to $20 \mathrm{~K}$ eliminate $3-15 \mathrm{~cm}^{-1}$ as possible sizes for $|J|$. In the [2Fe-2S] ferredoxins, $J$ values are often around $200 \mathrm{~cm}^{-1}$ [17] for two iron atoms separated by the sulfur atoms. We can expect the exchange couplings within the [4Fe-4S] cluster to be on this order; therefore, the $J$-couplings to the FMNH radical must be much smaller or they would compete with the intra-[4Fe-4S] cluster coupling and probably modify the $g$-values of the cluster. For this reason, we expect $|J|<50$ $\mathrm{cm}^{-1}$. A further estimate of the size of $J$ can be derived if we use the equation of Moriya [18], which states that the size of the anisotropic exchange interaction $(D)$ is around $\left(g-g_{\mathrm{e}}\right)^{2} J / 4$. If we estimate anisotropic exchange to be the sole cause of the zero field splitting parameter, $D$, then we can estimate the upper bound of $J$ from the $\mathrm{W}_{3} \mathrm{~A}_{1}$ data as approximately $50 \mathrm{~cm}^{-1}$ [10]. Therefore, the magnitude of $J$ is probably between 0.4 and 3 and 15 and $50 \mathrm{~cm}^{-1}$. If the magnitude of $J$ is greater than some $5 \mathrm{~cm}^{-1}$, then it must be positive, so that the triplet is the ground state, otherwise we would have to see a temperature dependence in the signal.

The zero-field parameter, $D$, can also result from a magnetic dipole-dipole interaction [12]. If $D$ results completely from dipole-dipole interaction, then the inter-site distance suggested from the data is between 3 and $5 \AA$. However, this calculation is made somewhat complicated and ambiguous, since most of the spin density on an $\mathrm{FMNH}^{\circ}$ radical is probably localized at the $\mathrm{N} 5$ and N10 positions [19] and that the magnetic field from a $[4 \mathrm{Fe}-4 \mathrm{~S}]$ cluster is not dipolar due to the fact that the four iron atoms in a cubane are antiferromagnetically coupled with their spins localized [17]. Calculations along this line have been made [10], and they represent an estimate of the order of magnitude of the inter-site distance between nearest neighbors of the triplet system if the $D$ value is from a magnetic dipolar interaction.

If one compares the numbers in the table among the three dehydrogenases, it is apparent that only the values of $D$ and $E$ differ significantly among the three. The $g$-values and Euler rotation angles are identical within error. Whether the zero-field splitting parameters result from anisotropic exchange or dipole-dipole interaction, they are very sensitive to distance. A trivial difference in the intersite distance could easily account for the data. On the other hand, the Euler angles are referenced to the [4Fe-4S] magnetic principal axis system. If the similarities in $g$-values indicate that the $[4 \mathrm{Fe}$ $4 \mathrm{~S}$ ] clusters are nearly identical in the three proteins, then the similarity in the Euler angles indicates that the geometry of the interacting system is almost identical in the three proteins. Therefore, the data support the interpretation that the $[4 \mathrm{Fe}$ $4 \mathrm{~S}^{1+}$-FMNH ${ }^{*}$ complex is a unique structure common to all three dehydrogenases studied here.

With regard to the origin of the distribution in the zero-field splitting parameters or ' $D$-strain', it can be shown [10] that the $D$-strain can be a mathematical consequence of ' $g$-strain', a distribution in the $g$-values, which we have obtained by fitting the uncoupled [4Fe-4S] EPR spectrum. By a multifrequency EPR study [10] of the signal in Fig. 1, we arrive at $\delta g / g=0.004$ where $\delta g$ is the standard deviation of $g_{x}=1.849$. If we apply Moriya's approximation to our own case (we estimate that $D$ is from anisotropic exchange [10]), then,

$D \cong 3(\Delta g)^{2} J / 4$

Assuming $J$ is constant and differentiating, we obtain

$\frac{\delta D}{D} \cong \frac{\delta(\Delta g)^{2}}{(\Delta g)^{2}}=2 \frac{\delta g}{\Delta g} \cong \frac{0.007}{0.035} \cong 0.2$

For all fits, the relative variance that gave the best fits was $\delta D / D=0.17$. Therefore, there appears to be no need for $J$-strain, since $g$-strain can account for the $D$-distribution. Furthermore, $g$-strain appears to be a ubiquitous component of the EPR spectra of frozen metalloproteins $[20,21]$ and has been recently interpreted as resulting from mechanical strains introduced by structural changes that are mainly at the exterior and hydrophilic regions of proteins. One can envision the broad wings in the $g=2$ region of Figs. 2, 4 and 5 as indicating a probable variation at the exterior of the protein. That Eqn. 5 accounts for the observed $D$-strain can be taken as an indication that the interaction responsible for $D$ is predominantly anisotropic exchange and not a dipole-dipole interaction, al- 
though these could be comparable. Because the exchange interaction is mediated by electron overlap between the two centers and this can occur through chemical bonds as well as through the vacuum, it is impossible for us to deduce a specific distance between the Fe-S center and the FMNH radical.

As described and discussed more extensively in Ref. 6, there are differences on addition of substrate in the behavior of the dimethylamine dehydrogenase studied and the two trimethylamine dehydrogenases. Whereas the triplet signal of the dimethylamine dehydrogenase simply rises in intensity as reduction progresses, the triplet signals of the trimethylamine dehydrogenases are not only intensified but the splitting between the lines increases. This is most pronounced with trimethylamine dehydrogenase of $\mathrm{W}_{3} \mathrm{~A}_{1}$ (cf. Fig. 1 of Ref. 7 and Fig. 4 of Ref. 8). The same observation is made in experiments where the enzyme is titrated with substrate and allowed to come to equilibrium. As an excess of substrate accumulates an analogous increase in total signal intensity and splitting of the outer lines is seen. We have proposed that this behavior is due to binding of excess substrate to the reduced form of the enzyme (Scheme 1 of Ref. 7) which leads to a conformational change to a more favorable geometry for development of the triplet state.

Recent crystallographic results on unreacted trimethylamine dehydrogenase crystals [22] have shown that the closest distance between the Fe-S cluster and the flavin is $6 \AA$. We hesitate to apply this result to our study on substrate-reduced trimethylamine dehydrogenase because the proposed mechanism for the protein probably requires some motion between the two centers. However, if the nearest approach of the flavin and Fe-S centers is also $6 \AA$ in the substrate-reduced protein, then the interaction between the centers is predominantly exchange rather than magnetic dipole-dipole.

\section{Acknowledgments}

This work was supported in part by the U.S. Public Health Service, GM 32785 (R.H.S.), GM12394 (H.B.), HL16251 (T.P.S.) and Research
Cancer Award 5-K06-GM-18442 (H.B.), by the National Science Foundation, PCM 81-19609 (T.P.S.) and by the Veterans Administration (T.P.S.). Our deepest gratitude goes to Dr. Daniel J. Steenkamp for his support and helpful discussions.

\section{References}

1 Steenkamp, D.J. and Mallinson, J. (1976) Biochim. Biophys. Acta 429, 705-719

2 Steenkamp, D.J. and Singer, T.P. (1978) Biochem. J. 169. $361-369$

3 Steenkamp, D.J., Beinert, H., McIntire, W. and Singer, T.P. (1978) in Mechanisms of Oxidizing Enzymes (Singer, T.P. and Ondarza, R.N., eds.) Vol. 1, pp. 127-141, Elsevier/ North-Holland, Amsterdam

4 Steenkamp, D.J. and Kennedy, W.C. (1980) in Flavins and Flavoproteins (Yagi, K. and Yamano, T., eds.), pp. 265-275, University Park Press, Baltimore

5 Singer, T.P., Steenkamp, D.J., Kenney, W.C. and Beinert, H. (1980) in Flavins and Flavoproteins (Yagi, K. and Yamano, T., eds.), pp. 277-287. University Park Press. Baltimore

6 Steenkamp, D.J. and Beinert, H. (1982) Biochem. J. 207. 233-239

7 Steenkamp, D.J. and Beinert, H. (1982) Biochem. J. 207. 241-252

8 Beinert, H., Shaw, R.W., Steenkamp, D.J., Singer, T.P., Stevenson, R., Dunham, W.R. and Sands, R.H. (1982) in Flavins and Flavoproteins (Massey, V. and Williams, C.H., eds.), Vol. 21, pp. 727-735. Elsevier/North-Holland, New York

9 Strong, L.H. (1976) Ph.D. thesis, University of Michigan

10 Stevenson, R.C. (1982) Ph.D. thesis, University of Michigan

11 Reid, J.A. (1976) Ph.D. thesis University of Michigan

12 Stevenson, R.C. (1984) J. Magn. Reson. 57, 24-42

13 Hearshen, D.O. (1983) Ph.D. thesis, University of Michigan

14 Schepler, K.L., Dunham, W.R., Sands, R.H., Fee, J.A. and Abeles, R.H. (1975) Biochim. Biophys. Acta 397, 510-518

15 Ruzicka, F.J., Beinert, H., Schepler, K.L., Dunham, W.R. and Sands, R.H. (1976) Proc. Natl. Acad. Sci. USA 72, 2886-2890

16 Schepler, K.L. (1975) Ph.D. thesis, University of Michigan

17 Sands, R.H. and Dunham, W.R. (1975) Q. Rev. Biophys. 7, 443-504

18 Moriya, T. (1960) Phys. Rev. 120, 91-98

19 Platenkamp, R.J., Palmer, M.H. and Visser, A.J.W.G. (1980) J. Mol. Phys. 67, 45-64

20 Hagen, W.R., Hearshen, D.O., Sands, R.H. and Dunham, W.R. (1985) J. Magn. Reson. 61, 220-232

21 Hagen, W.R., Hearshen, D.O., Harding, R.J. and Dunham, W.R. (1985) J. Magn. Reson. 61, 233-244

22 Mathews, F.S., Lim, L.W. and Shamala, N.S. (1984) in Flavins and Flavoproteins (Bray, R.C., Engel, P. and Mayhew, S.G., eds.), pp. 233-246, W. de Gruyter, Berlin 